\section{Toward the on-line analysis of raw bioelectric signals: The case of EEG*}

\author{
D. G. WHITMORE and L. R. GORDON \\ University of Vermont, Burlington, Vermont 05401
}

Human physiological measures such as EKG, REM (rapid eye movements), GSR, and EEG can be useful to both physiologists and psychologists, although the ways such data are treated by each group differ. In the case of EEG, the physiologist uses electrical signals from the brain as clues to brain function, and the analyses used are complex. Typically, auto- and cross-correlations, Fourier analysis, and signal averaging are employed. The psychologist, on the other hand, is interested in EEG as a descriptive measure for states such as motivation, emotion, or arousal which are primarily of psychological interest. Psychologists have used less elaborate analyses of EEG, since the goal of this research as a whole is to describe states of EEG and to correlate them with other behavioral elements. In the past, several techniques have been widely used by psychologists to extract descriptive information from EEG polygraph paper records. These have consisted of "a subjective analysis by an experienced electroencephalographer," exacting measurements with calipers of the paper record or abstracting salient features of the record while ignoring others. These techniques have been basically unsatisfactory in that they are slow,

*This work was sponsored in part by USAF Office of Scientific Research Grant No. F44620-69-C. imprecise, and fail to give sufficient feedback to the $E$. In our work in circadian rhythms and sensory deprivation, large amounts of this data had to be processed in the course of an experiment, and it became not only unsatisfactory but impossible to treat data with hand techniques.

The small, dedicated digital computer was an ideal choice for reducing this large volume of data on-line during an experimental session. Its speed and flexibility can give a rapid summary of global bioelectric activity including EEG to the E according to any sampling schedule specified by the experimental design employed. A PDP-12 computer with $8 \mathrm{~K}$ of core memory was used, and special software was developed to perform the analysis. Conventional software for EEG analysis was available to analyze data by Fourier analysis, correlations, etc., but these techniques were not compatible with our previous work, dealt with a small, rather than a global, time slice of the signals, and violated some of the mathematical assumptions of the analyses themselves. Leaving interpretation of EEG data processed in this way to neurophysiologists, we set out to develop software which would present a simpler, yet accurate, picture to the psychological E.

At present, we have completed the EEG frequency portion of a comprehensive package. The basic operating principle of the program is the timing of a single ac wave from an artifact-free record which is carried out partly by hardware and partly by software. This time period is, of course, related to the frequency. Over an extended sampling interval of from 1 to $3 \mathrm{~min}$, a location in core memory corresponding to this period will be incremented by one each time a wave of that period is observed. This procedure of using the period as an index to increment a location is extremely fast and permits a minimum of computation along with a high clock rate $(1 \mathrm{kHz})$, resulting in maximum frequency resolution. The elaborate calculations required by more complex analyses may cause events to be inaccurately measured if computation was underway when data was ready for processing.

Acquisition may be interrupted at any time and a summary printed out, a CRT display obtained, or core cleared for the next sample. The printed summary consists of the relative proportions between and the average frequency within the alpha, beta, and theta EEG bands. Our frequency definitions correspond to the traditional ones but can readily be changed by software.

The EEG frequency program described above will become part of a comprehensive software package for the analysis of several bioelectric measures. Eventually, EEG amplitude, average REM activity, and average heart-rate analyses will be added. Provision will be made for the automatic adjustment of signal gains via a computer-controlled operational amplifier. The completed system will provide a concise summary of several parameters from complex and voluminous physiological data on-line during an experimental session. 